\title{
Regulation of Amidase Synthesis by Pseudomonas aeruginosa 8602 in Continuous Culture
}

\author{
By ANNA BODDY, PATRICIA H. CLARKE, \\ MARGARET A. HOULDSWORTH \\ Department of Biochemistry \\ AND M. D. LILLY \\ Biochemical Engineering Section, Department of Chemical Engineering, \\ University College, London
}

(Accepted for publication 22 February 1967)

SUMMARY

\begin{abstract}
Pseudomonas aeruginosa 8602 was grown in continuous culture in minimal salts medium with $10 \mathrm{~mm}$-succinate as carbon source. Changes in bacterial concentration and amidase activity were measured when the carbon source in the ingoing medium was changed to $10 \mathrm{mM}$-succinate plus $10 \mathrm{mM}$-acetamide. At low dilution rates $\left(D=0.2 \mathrm{hr}^{-1}\right)$ amidase synthesis occurred rapidly with little or no lag. At higher dilution rates the lag increased until at the highest dilution rate tested $\left(D=0.76 \mathrm{hr}^{-1}\right)$ almost no amidase had been synthesized $4 \mathrm{hr}$ after the change of medium. It was concluded that at the highest dilution rates amidase synthesis was subject to catabolite repression by high intracellular concentrations of succinate and its metabolites. Severe repression of amidase synthesis was accompanied by incomplete utilization of acetamide for growth.

Periodic oscillations were observed in amidase activity and in bacterial concentration both during the transition period after the change of medium and when the culture had reached the new steady state. These oscillations occurred at all dilution rates and oscillations in amidase activity were also found when the non-substrate inducer $N$-acetylacetamide was supplied with 20 mM-succinate.
\end{abstract}

\section{INTRODUCTION}

Pseudomonas aeruginosa 8602 produces an aliphatic amidase (acylamide amidohydrolase E.C. 3.5.1.4) which is regulated by induction and catabolite repression. Cultures growing exponentially in succinate medium synthesize amidase at a constant differential rate after a lag of about one generation from the time of addition of substrate and non-substrate inducers. Amidase synthesis in pyruvate medium starts after a lag of only $\mathbf{0 . 2 5}$ of a generation and reaches a higher specific activity. The addition of $10 \mathrm{~mm}$-succinate to exponentially growing cultures in pyruvate medium, induced by the non-substrate inducer $N$-acetylacetamide, results in almost complete repression of amidase synthesis (Brammar \& Clarke, 1964; Brammar, Clarke \& Skinner, 1967). Amidase induction by acetamide or $N$-acetylacetamide in carbonstarved bacteria is also repressed by succinate and the extent of repression is dependent on the relative concentrations of succinate and inducer. The effect does not appear to be due to direct competition between inducer and co-repressor of the type found 


\section{A. BODDY, P. H. CLARKE, M. A. HOULDSWORTH AND M. D. LILLY}

for repression of amidase synthesis by amide analogues such as cyanoacetamide (Clarke \& Brammar, 1964).

The study of enzyme regulation during growth has been carried out almost entirely with organisms growing exponentially in batch culture. However, Gorini (1960) examined repression of the enzymes of the arginine biosynthetic pathway in continuous culture and showed that at certain dilution rates glutamic acid could relieve the repression, by arginine, of ornithine transcarbamylase synthesis (Gorini, 1963). McFall \& Mandelstam (1963) studied catabolite repression of three inducible enzymes produced by Escherichia coli, $\beta$-galactosidase, serine deaminase and tryptophanase in both batch and continuous culture. In a different type of study, Tempest \& Herbert (1965) examined the effect of dilution rate and carbon substrate on the metabolic activity of Torula utilis.

In batch culture, glucose represses the synthesis of certain enzymes so that the organisms do not utilize those substrates for growth until the glucose has almost disappeared from the medium. Klebsiella aerogenes exhibits typical diauxic growth in batch cultures containing glucose + maltose or glucose +lactose. The 'glucose effect' can also be observed in continuous culture in media containing these pairs of sugars. Growth, and the extent of utilization of the sugars, is related to the culture dilution rate (Baidya, Webb \& Lilly, 1966; Harte, 1965). We considered that these results could be interpreted in terms of catabolite repression by glucose of the enzymes required to utilize the second sugar, and that this technique of growth on two different carbon compounds in continuous culture could be used to study enzyme regulation in other systems.

Pseudomonas aeruginosa has been grown in continuous culture in succinate medium at various dilution rates, and the rates of growth and amidase synthesis have been measured following a change of medium to succinate + acetamide. A brief report of part of this work has appeared previously (Boddy, Clarke \& Lilly, 1966).

\section{METHODS}

Organism. The strain used was Pseudomonas aeruginosa 8602 (Brammar et al. 1967). It was maintained on slopes of lemco agar, subcultured weekly and stored at $4^{\circ}$.

Media. The minimal salts medium contained (g./1.): $\mathrm{K}_{2} \mathrm{HPO}_{4}, 12 \cdot 5 ; \mathrm{KH}_{2} \mathrm{PO}_{4}, 3 \cdot 8$; $\left(\mathrm{NH}_{4}\right)_{2} \mathrm{SO}_{4}, 1.0 ; \mathrm{MgSO}_{4} .7 \mathrm{H}_{2} \mathrm{O}, 0.1$; trace element solution, $5 \mathrm{ml}$. (Kelly \& Clarke, 1962). Succinate (to give a final concentration of either 10 or $20 \mathrm{~mm}$ ) was added to the medium in 201 . Pyrex glass aspirators before autoclaving at $121^{\circ}$ for $2 \mathrm{hr}$.

Acetamide and $\mathrm{N}$-acetylacetamide were sterilized by Seitz filtration and added aseptically to the minimal salts + succinate media to give final concentrations of $20 \mathrm{~mm}$ and $10 \mathrm{~mm}$ respectively. The continuous culture experiments were all carried out under conditions in which growth was limited by the carbon source.

Culture equipment. An inoculum (70 ml.), grown on minimal salts + succinate medium, was blown from the seed vessel into a 51 . vessel containing 31 . of growth medium at a controlled temperature of $37^{\circ}$. The $\mathrm{pH}$ value of the medium was controlled at $7 \cdot 2$ by the addition of $\mathrm{N}-\mathrm{HCl}$. Air was supplied through the bottom of the vessel at $11 . / \mathrm{min}$. The carbon dioxide content of the effluent gas was measured by passing it through an infra-red analyser (type SBK, Infra-red Development Co. Ltd.). Foaming was prevented by the addition of polypropylene glycol (100 p.p.m.) to the medium. 
During continuous operation the growth medium was fed from the 201 . aspirator into the 51 . vessel by a peristaltic pump constructed in this laboratory. Medium left the vessel by overflowing down a standpipe inserted through the bottom of the vessel. Samples (about $5 \mathrm{ml}$.) were withdrawn from the medium through a narrow-bore tube leading to a collecting bottle. The sample was diluted, chloramphenicol added, and then part used for determination of the bacterial concentration. The remainder was cooled rapidly in an ice-bath and assayed for amidase within a few minutes.

Dry weight of bacteria. The optical extinction of diluted bacterial suspensions were measured at $670 \mathrm{~m} \mu$ with a Unicam SP 600 spectrophotometer. A standard curve was used to convert the readings to dry weights (mg./ml.).

Enzyme assays. Amidase was measured using the hydroxamate method for transferase activity described by Brammar \& Clarke (1964). For samples with high enzyme activity, $\mathbf{0 . 1} \mathrm{ml}$. of the diluted bacterial suspension was used in each assay mixture which was incubated at $37^{\circ}$ for $5 \mathrm{~min}$. The amidase specific activities were calculated as $\mu$ moles acethydroxamate formed $/ \mathrm{mg}$. dry weight of bacteria $/ \mathrm{min}$.

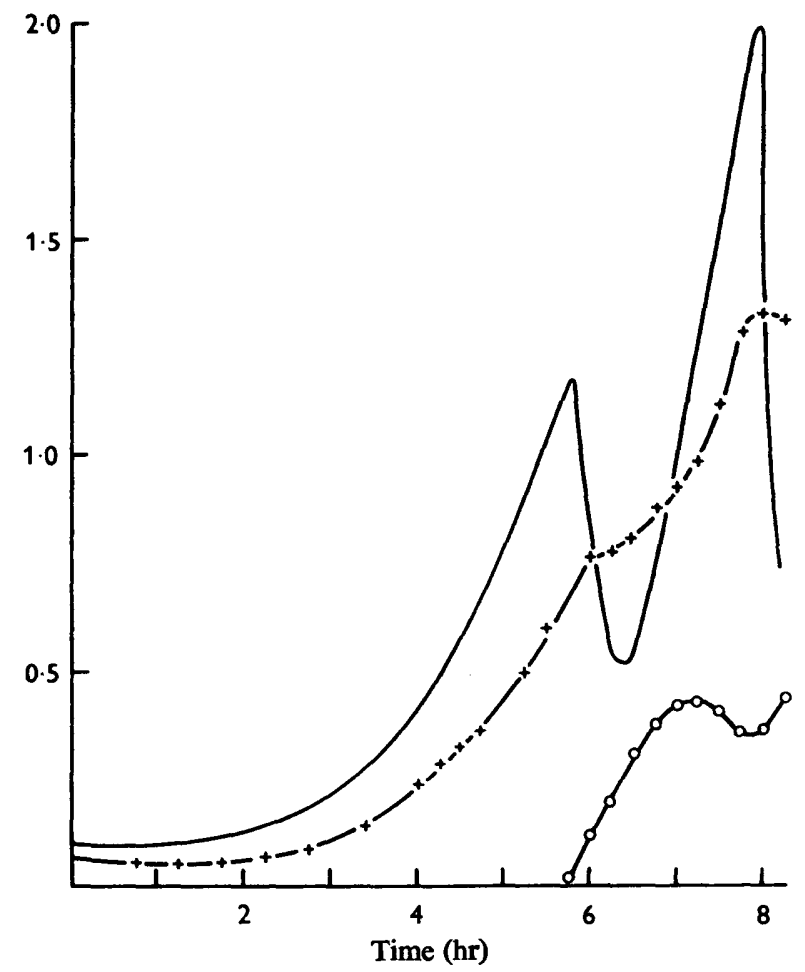

Fig. 1. Growth of Pseudomonas aeruginosa 8602 with $10 \mathrm{~mm}$-succinate: + , bacterial concentration $\left(E_{870 \mathrm{~m} \mu}\right) ; O$, Amidase specific activity $\left(\times 10^{-1}\right) ;-$, carbon dioxide in exit gas $(\%)$.

\section{RESULTS}

Growth on succinate and acetamide in batch culture

Before starting the continuous culture experiments the growth of Pseudomonas aeruginosa 8602 in the 51 . vessel was examined in batch culture in a medium containing $10 \mathrm{~mm}$-succinate $+20 \mathrm{~mm}$-acetamide (Fig. 1). The growth followed a typical diauxie 
with succinate being consumed in the first growth phase. The presence of a diauxic growth lag was confirmed by the bimodal curve for the carbon dioxide in the effluent gas. Amidase synthesis began during the diauxic lag and was followed soon afterwards by renewed bacterial growth on the acetate which had been formed. At the end of the second growth phase the amidase specific activity had reached about 5 .

\section{Growth on succinate and acetamide in continuous culture}

Growth on succinate and succinate + acetamide in continuous culture was examined at various dilution rates. Initially, the culture was allowed to reach a steady state on a medium containing $10 \mathrm{~mm}$-succinate. The incoming medium then was changed to one containing $10 \mathrm{~mm}$-succinate $+20 \mathrm{~mm}$-acetamide which would allow the bacterial concentration to double if completely utilized. The synthesis of amidase and the change in bacterial concentration were followed during the shift to the new steady-state condition. The results of an experiment carried out at a low dilution rate $\left(0.22 \mathrm{hr}^{-1}\right)$ are shown in Fig. 2. At this dilution rate, amidase synthesis started very soon after the change to the succinate + acetamide medium. The amidase specific activity continued to increase over several hours but with peaks of higher activity at more or less regular intervals of 45 to $50 \mathrm{~min}$. Smaller but nevertheless significant fluctuations were observed in the bacterial concentration as it rose towards its new steady state. Three identical experiments were done at this dilution rate and in each case the curves for amidase and bacterial concentration were similar. Further measurements were made on the following day, by which time both the amidase and the bacterial concentrations had reached new mean steady-state values and it was found that regular oscillations were still occurring. The maximum amidase specific activity was about 100 .

The results of a similar experiment done at a higher dilution rate $\left(0.48 \mathrm{hr}^{-1}\right)$ are shown in Fig. 3. Again, oscillations were observed in both amidase and bacterial concentration and these continued unabated on the following day. The periodicity appeared to be about the same as at the lower dilution rate but the increase in amidase specific activity was much slower and the maximum value reached was only about 50 in the new steady state compared to the value of about 100 obtained at the lower dilution rate.

The effect of dilution rate on the rate of amidase synthesis during the transition period following the change to a medium containing succinate + acetamide is shown more clearly in Fig. 4. This gives the results for similar experiments done at four different dilution rates and it can be seen that amidase synthesis is severely repressed at the higher dilution rates. At the highest dilution rate investigated $\left(0.76 \mathrm{hr}^{-1}\right)$ the amidase activity was only very slightly above the basal level on succinate alone. It can be seen from Fig. 4 that oscillations in amidase activity occur at all these dilution rates although the specific activities differ widely.

\section{Amidase induction by $\mathrm{N}$-acetylacetamide in continuous culture}

Comparable experiments to those already described were carried out using the nonsubstrate inducer, $\mathrm{N}$-acetylacetamide. After allowing the culture to reach a steady state on a medium containing $10 \mathrm{~mm}$-succinate the medium was changed to one containing $20 \mathrm{~mm}$-succinate $+10 \mathrm{~mm}-N$-acetylacetamide. The changes in amidase specific activities at various dilution rates are shown in Fig. 5. Once again it was found that amidase synthesis was repressed at the higher dilution rates. The specific activities 
obtained with this non-substrate inducer were much lower than with acetamide. Periodic oscillations in amidase activity were less apparent but could certainly be detected in the curves for the two lower dilution rates $\left(0.17\right.$ and $\left.0.51 \mathrm{hr}^{-1}\right)$.

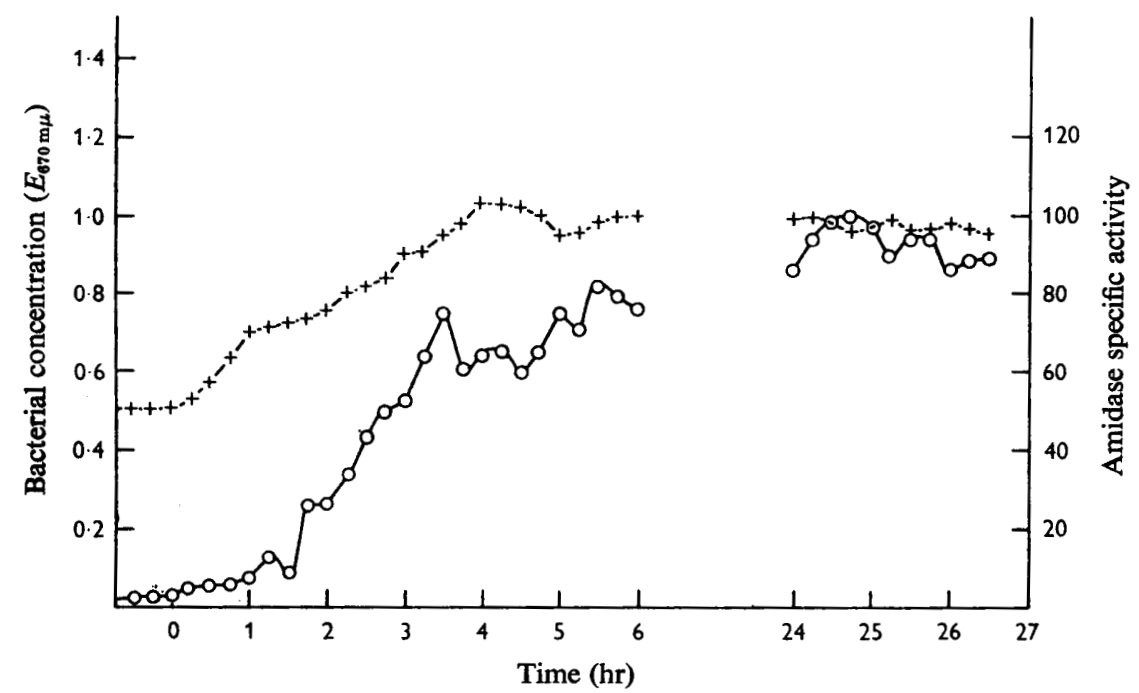

Fig. 2. Amidase synthesis by Pseudomonas aeruginosa 8602 in continuous culture $(D=$ $\left.0.22 \mathrm{hr}^{-1}\right):+$, bacterial concentration $\left(E_{670 \mathrm{~m} \mu}\right) ; 0$, amidase specific activity. At zero time the ingoing medium was changed from $10 \mathrm{~mm}$-succinate to $10 \mathrm{~mm}$-succinate $+20 \mathrm{~mm}$ acetamide.

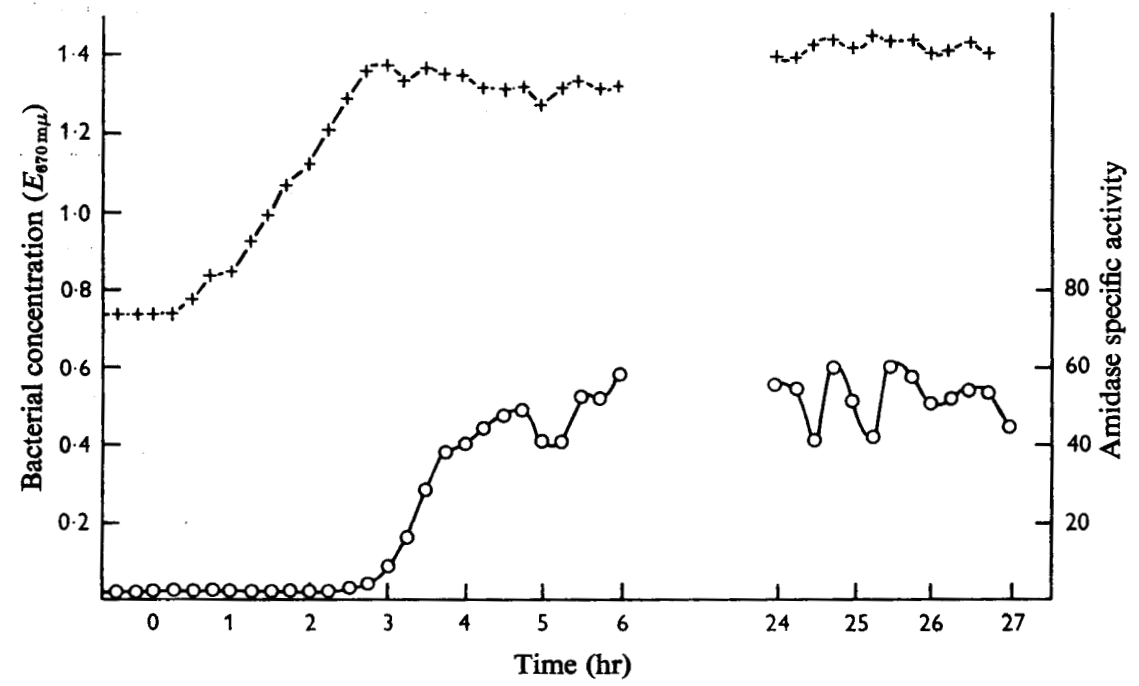

Fig. 3. Amidase synthesis by Pseudomonas aeruginosa 8602 in continuous culture $(D=$ $\left.0.48 \mathrm{hr}^{-1}\right):+$, bacterial concentration $\left(E_{670 \mathrm{~m} \mu}\right) ; O$, amidase specific activity. At zero time the ingoing medium was changed from $10 \mathrm{~mm}$-succinate to $10 \mathrm{~mm}$-succinate $+20 \mathrm{~mm}$ acetamide. 


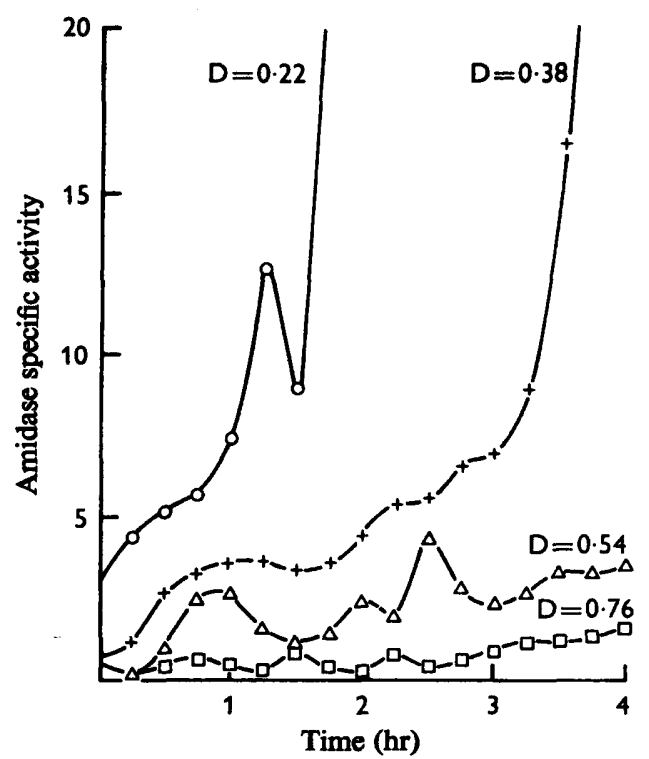

Fig. 4

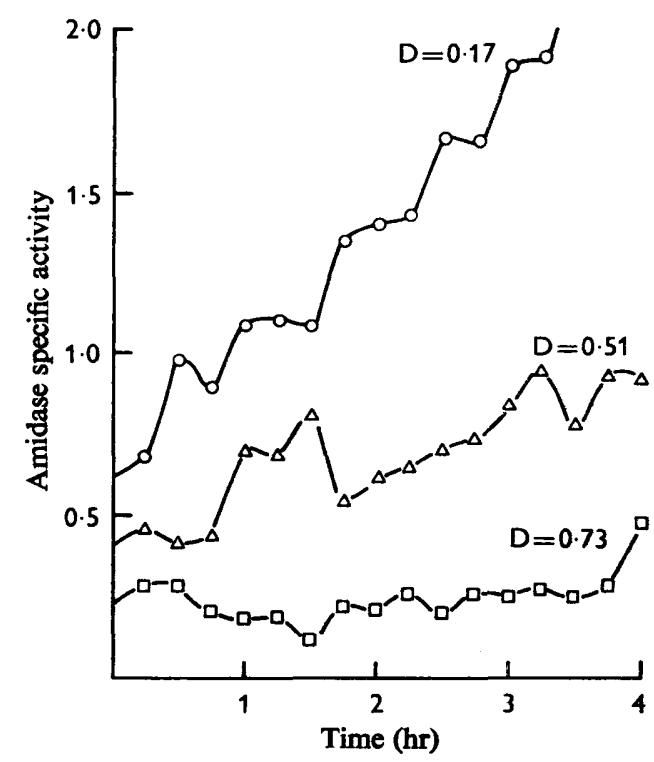

Fig. 5

Fig. 4. Effect of dilution rate, $D\left(\mathrm{hr}^{-1}\right)$, on amidase synthesis by Pseudomonas aeruginosa growing with succinate + acetamide. At zero time the ingoing medium was changed from $10 \mathrm{~mm}$-succinate to $10 \mathrm{~mm}$-succinate $+20 \mathrm{~mm}$-acetamide.

Fig. 5. Effect of dilution rate, $D\left(\mathrm{hr}^{-1}\right)$, on amidase synthesis by Pseudomonas aeruginosa growing with succinate in the presence of the non-substrate inducer, $N$-acetylacetamide. At zero time the ingoing medium was changed from $10 \mathrm{~mm}$-succinate to $20 \mathrm{~mm}$-succinate+ $10 \mathrm{mM}-N$-acetylacetamide.

\section{DISCUSSION}

The results in this study confirm that amidase induction in Pseudomonas aeruginosa 8602 is repressed by metabolites formed during succinate metabolism. In the batchculture experiment amidase synthesis was completely repressed until the succinate had disappeared from the medium, and even then the maximum activity reached was not very high. This was partly because the inducer acetamide is rapidly hydrolysed by the newly formed enzyme and disappears from the medium. It had been shown previously that when acetamide is added to an exponentially growing culture amidase induction falls off as the acetamide is hydrolysed. It is also probable that amidase synthesis is repressed by intermediates of acetate metabolism, since it is known that acetate represses amidase synthesis in exponentially growing cultures induced with either acetamide or $N$-acetylacetamide (Brammar \& Clarke, 1964). It is nevertheless possible to induce amidase synthesis under conditions in which succinate is still present in the medium as the major carbon source. The rates of amidase synthesis and the specific activities reached are much less in succinate medium than in pyruvate medium, both for induced and constitutive strains (Brammar et al. 1967). This suggests that succinate is a good source of the effective repressing metabolite and is similar to the observation that $\beta$-galactosidase in Escherichia coli is more readily induced in glycerol than in glucose medium (Loomis \& Magasanik, 1964). Clarke \& Brammar (1964) found that amidase could be induced in carbon-starved organisms from batch 
culture by much lower concentrations of acetamide and $N$-acetylacetamide, with little or no lag. Under these conditions the intracellular concentration of intermediary metabolites would be expected to be minimal.

In continuous culture at low dilution rates the growth rate of the bacteria is correspondingly low. Growth is carbon-limited, intermediary metabolites are fully utilized and the internal carbon-pool depleted. When acetamide is introduced into the medium the conditions resemble those in carbon-starved batch culture, the conditions are favourable for amidase induction and the rate of synthesis will be regulated by the supply of inducer. The high amidase specific activity obtained under these conditions is similar to that obtained in batch culture in pyruvate medium. The specific activity of the pure enzyme is about 2000 (P. R. Brown \& P. H. Clarke, unpublished) and at the low dilution rates on succinate + acetamide medium the amidase constitutes about $5 \%$ of the dry weight of the bacterial cells.

At higher dilution rates and correspondingly higher growth rates, the pool of intermediary metabolites is less depleted. The balance between inducer and catabolite co-repressor is shifted in favour of repression, until at the highest dilution rate examined amidase synthesis is almost completely repressed. Under these severely repressing conditions the amount of amidase formed is insufficient to hydrolyze all the incoming acetamide, so that it cannot be fully utilized for bacterial growth. The catabolite repression by succinate appears to be partially overcome after a certain period of time. During the change from one steady state on succinate to the new steady state on succinate + acetamide the bacterial growth rate increases, resulting in a higher level of catabolite co-repressor and a low rate of amidase synthesis. As the new steady state is approached the growth rate gradually returns to its original level with a corresponding reduction in the concentration of intermediary metabolites and a decrease in repression of amidase synthesis. It is therefore a period in which there is an adjustment in the relative concentrations of intermediary metabolites. It has been suggested by Moses \& Prevost (1966) that metabolic rearrangements occur during the transient catabolite repression of $\beta$-galactosidase synthesis in Escherichia coli.

In batch culture, acetamide is less effective as an inducer than $N$-acetylacetamide because it is consumed as soon as an appreciable amount of amidase has been formed. In carbon-starved bacteria from batch culture, and in continuous culture where the organisms are being continually presented with new acetamide, the reverse is the case. When amidase synthesis in continuous culture was examined under conditions of gratuity with $\mathrm{N}$-acetylacetamide as non-substrate inducer, although the inducer concentration was rising faster at the higher dilution rates, the increase in the concentration of intermediary metabolites prevented induction. It was not possible to do prolonged experiments in continuous culture with $N$-acetylacetamide, as this compound is not sufficiently stable, so all these experiments were terminated after $4 \mathrm{hr}$.

A further effect observed at all dilution rates was the periodic oscillation of amidase activity in the culture accompanied by a less marked oscillation in bacterial concentration. Although the possibility that these oscillations were caused by physical factors cannot be entirely ruled out, we have carried out exhaustive tests on such parameters as mixing, $\mathrm{pH}$ and temperature control and are satisfied that these oscillations are not artifacts of the culture system. Certainly, in other series of experiments in the same type of culture vessel, we have not observed fluctuations in bacterial concentration to the extent found in these experiments. 
These fluctuations suggest that there may be some degree of cell synchrony in these experiments. It is difficult to measure accurately the periodicity of the oscillations in bacterial concentration but it appears to be slightly longer than that for amidase concentration. We believe that this effect on bacterial concentration is a reflexion of oscillations in the concentration of a growth-limiting enzyme or enzyme systems. The oscillations in amidase concentration should only reflect changes in the concentration of the inducer or catabolite co-repressor and need not be in phase with or have the same periodicity as the changes in bacterial concentration. With acetamide as the inducer it was not possible to decide whether the changes in enzyme concentration were the result of variations in the level of the inducer or the catabolite co-repressor or both. These oscillations were still present when the non-substrate inducer $\mathrm{N}$ acetylacetamide was used, so that it is unlikely that the oscillations observed with acetamide are the result of changes in the concentration of the substrate inducer caused by varying rates of acetamide hydrolysis.

It was quite possible that the oscillations in amidase concentration observed during the transition to the new steady state were caused by the sudden change in the incoming medium. However, these oscillations were still occurring to about the same extent on the following day, indicating that the system was probably undamped and likely to continue indefinitely.

This periodic oscillation of amidase activity is of considerable interest in view of the theoretical predictions by Goodwin $(1963,1966)$ that oscillations in enzyme synthesis could occur in systems controlled by feedback mechanisms and might be found in continuous culture. Oscillations in the extracellular pyruvate concentration have been observed by Sikyta \& Slezak (1965) for continuous cultures of $E$. coli growing on lactate and this is probably a reflexion of changes in enzyme levels in the cells. Our results demonstrate (in continuous culture) the dynamic situation which exists between the induction of an enzyme by its substrate analogues or substrate and its repression by related metabolites. It seems likely that the observed oscillations are a further expression of the catabolite repression which exists in this system. The problem as to why there should be this metabolic synchrony in a bacterial population remains to be solved.

\section{REFERENCES}

BAIDYA, T. K. N., WEBB, F. C. \& LiLLY, M. D. (1967). Utilization of mixed sugars in continuous fermentation. Biotech. Bioeng. (in the Press).

Boddy, A., Clarke, P. H. \& Lilly, M. D. (1966). Periodic changes in amidase synthesis by Pseudomonas aeruginosa in continuous culture. J. gen. Microbiol. 45, ii.

Brammar, W. J. \& Clarke, P. H. (1964). Induction and repression of Pseudomonas aeruginosa amidase. J. gen. Microbiol. 37, 307.

Brammar, W. J., Clarke, P. H. \& Skinner, A. J. (1967). Biochemical and genetic studies with regulator mutants of the Pseudomonas aeruginosa 8602 amidase system. J. gen. Microbiol. $47,87$.

Clarke, P. H. \& Brammar, W. J. (1964). Regulation of bacterial enzyme synthesis by induction and repression. Nature, Lond. 203, 1153.

GoodwIN, B. C. (1963). Temporal Organization in Cells. London: Academic Press.

GoodwIN, B. C. (1966). Entrainment model for timed enzyme syntheses in bacteria. Nature, Lond. $209,479$.

GorinI, L. (1960). Antagonism between substrate and repressor in controlling the formation of a biosynthetic enzyme. Proc. natn. Acad. Sci., U.S.A. 46, 682.

GoRINI, L. (1963). Control by repression of a biochemical pathway. Bact. Rev. 27, 182.

HARTE, M. J. (1965). Utilisation of sugars in continuous fermentation. Ph.D. thesis, University of London. 
Kelly, M. \& Clarke, P. H. (1962). An inducible amidase produced by a strain of Pseudomonas aeruginosa. J. gen. Microbiol. 27, 305.

LOOMIS, W. F. \& MAGASANIK, B. (1964). The relation of catabolite repression to the induction system for $\beta$-galactosidase in Escherichia coli. J. molec. Biol. 8, 417.

MCFALL, E. \& MANDELSTAM, J. (1963). Specific metabolic repression of three individual enzymes in Escherichia coli. Biochem. J. 89, 391.

Moses, V. \& PREVOST, C. (1966). Catabolic repression of $\beta$-galactosidase synthesis in Escherichia coli. Biochem. J. 100, 336.

Sikyta, B. \& SlezAK, J. (1965). A periodic phenomenon in regulation of pyruvate biosynthesis in Escherichia coli B. Biochim. biophys. Acta 100, 311.

TEMPEST, D. W. \& HERBERT, D. (1965). Effect of dilution rate and growth-limiting substrate on the metabolic activity of Torula utilis cultures. J. gen. Microbiol. 41, 143. 